\title{
NEW EXAMPLES OF WILLMORE SUBMANIFOLDS IN THE UNIT SPHERE VIA ISOPARAMETRIC FUNCTIONS, II
}

\author{
CHAO QIAN, ZIZHOU TANG, AND WENJIAO YAN ${ }^{\dagger}$
}

Dedicated to Professor Banghe Li on his 70-th birthday.

\begin{abstract}
This paper is a continuation and wide extension of TY]. In the first part of the present paper, we give a unified geometric proof that both focal submanifolds of every isoparametric hypersurface in spheres with four distinct principal curvatures are Willmore. In the second part, we completely determine which focal submanifolds are Einstein except one case.
\end{abstract}

\section{INTRODUCTION}

Let $x: M^{n} \rightarrow S^{n+p}$ be an immersion from an $n$-dimensional compact manifold to an $(n+p)$-dimensional unit sphere $S^{n+p}$. Denote by $h$ the second fundamental form of $x, S$ the norm square of $h$, and $H$ the norm of the mean curvature vector, respectively. Then $M^{n}$ is called a Willmore submanifold in $S^{n+p}$ if it is an extremal submanifold of the Willmore functional, which is a conformal invariant ( $c f$. Wan]):

$$
W(x)=\int_{M^{n}}\left(S-n H^{2}\right)^{\frac{n}{2}} d v .
$$

An equivalent condition for $M^{n}$ to be Willmore was given in [GLW], [PW]. In particular, when $M^{n}$ is a minimal submanifold in $S^{n+p}$ with constant $S$, under a field of local orthonormal basis $\left\{e_{A}\right\}(1 \leq A \leq n+p)$ for $T S^{n+p}$, in which $\left\{e_{i}\right\} \in T M$ $(1 \leq i \leq n)$ and $\left\{e_{\alpha}\right\} \in T^{\perp} M(n+1 \leq \alpha \leq n+p)$, their criterion for Willmore reduces to be:

$$
\text { for any } \alpha, \sum_{i, j=1}^{n} R_{i j} h_{i j}^{\alpha}=0,
$$

where $R_{i j}$ is the Ricci tensor of $M^{n}, h_{i j}^{\alpha}$ is the component of $h$ with respect to $e_{i}, e_{j}$ and $e_{\alpha}$.

2000 Mathematics Subject Classification. 53A30, 53C42.

Key words and phrases. Willmore submanifold, isoparametric functions, focal submanifolds.

$\dagger$ the corresponding author.

The project is partially supported by the NSFC ( No.11071018) and the Program for Changjiang Scholars and Innovative Research Team in University. 
Based on the reduced criterion (1), in conjunction with the fact that the focal submanifolds of an isoparametric hypersurface in $S^{n+1}$ are minimal submanifolds of $S^{n+1}$ with constant $S$, we establish one of our main results as follows

Theorem 1.1. Both focal submanifolds of every isoparametric hypersurface in unit spheres with four distinct principal curvatures are Willmore.

Remark 1.1. This theorem extends widely the main result in [TY], where one focal submanifold of every isoparametric hypersurface of FKM-type was shown to be Willmore.

We need some preliminaries on isoparametric hypersurfaces. It is well known that an isoparametric hypersurface in a complete Riemannian manifold $N$ always comes as a family of parallel hypersurfaces, which are level hypersurfaces of an isoparametric function $f$, that is, a non-constant smooth function on $N$ satisfying

$$
\left\{\begin{array}{c}
|\nabla f|^{2}=b(f), \\
\Delta f=a(f),
\end{array}\right.
$$

where $\nabla f$ and $\Delta f$ are the gradient and Laplacian of $f$ on $N$, respectively, $b$ and $a$ are smooth and continuous functions on $\mathbb{R}$, respectively. The preimage of the global maximum (resp. minimum) of an isoparametric function $f$ is called the focal variety of $f$, denoted by $M_{+}$(resp. $M_{-}$), if nonempty. A fundamental structural result claimed by [Wan', proved in details by [GT], asserts that each focal variety of an isoparametric function is a minimal submanifold of $N$.

The equations in (2) mean that the parallel level hypersurfaces have constant mean curvatures. As is well known, an isoparametric hypersurface $M^{n}$ in the unit sphere $S^{n+1}$ actually has constant principal curvatures. Let $g$ be the number of distinct principal curvatures, which are denoted by $k_{i}\left(k_{1}>\ldots>k_{g}\right)$ with multiplicity $m_{i}(i=1, \ldots, g)$. A remarkable result proved by Münzner [Mun] states that $m_{i}=m_{i+2}$ (subscripts mod $g$ ) and the isoparametric function $f$ must be the restriction to $S^{n+1}$ of a homogeneous polynomial $F: \mathbb{R}^{n+2} \rightarrow \mathbb{R}$ of degree $g$ satisfying the Cartan-Münzner equations:

$$
\left\{\begin{array}{l}
|\nabla F|^{2}=g^{2}|x|^{2 g-2} \\
\Delta F=\frac{m_{2}-m_{1}}{2} g^{2}|x|^{g-2},
\end{array}\right.
$$

where $\nabla F$ and $\Delta F$ are the gradient and Laplacian of $F$ on $\mathbb{R}^{n+2}$. The polynomial $F$ is called the Cartan-Münzner polinomial, its restriction $f=\left.F\right|_{S^{n+1}}$ takes values in $[-1,1]$ on $S^{n+1}$. The focal submanifolds $M_{+}:=f^{-1}(1)$ and $M_{-}:=f^{-1}(-1)$ are in fact minimal submanifolds of $S^{n+1}$ with respective codimensions $m_{1}+1$ and $m_{2}+1$, whose second fundamental forms are both of constant length ( $c f$. [CR]).

As a corollary of the criterion (1), every n-dimensional Einstein manifold minimally immersed in the unit sphere $S^{n+p}$ is a Willmore submanifold. Then a natural problem 
arises: is every focal submanifold Einstein? According to the concluding remark of [TY], there are only few focal submanifolds $M_{+}$of FKM-type being possibly Einstein. As another main result of this paper, we give a complete resolution of this problem for the focal submanifolds of every isoparametric hypersurface in $S^{n+1}$ with four distinct principal curvatures, except the open case $\left(m_{1}, m_{2}\right)=(7,8)$.

To state Theorem 1.2 clearly, we recall the construction of isoparametric functions of FKM-type. For a symmetric Clifford system $\left\{P_{0}, \cdots, P_{m}\right\}$ on $\mathbb{R}^{2 l}$, i.e. $P_{i}$ 's are symmetric matrices satisfying $P_{i} P_{j}+P_{j} P_{i}=2 \delta_{i j} I_{2 l}$, Ferus, Karcher and Münzner ([FKM]) constructed a polynomial $F$ (called FKM-type isoparametric polynomial) of degree 4 on $\mathbb{R}^{2 l}$ :

$$
\begin{gathered}
F: \quad \mathbb{R}^{2 l} \rightarrow \mathbb{R} \\
F(x)=|x|^{4}-2 \sum_{i=0}^{m}\left\langle P_{i} x, x\right\rangle^{2},
\end{gathered}
$$

which satisfies the Cartan-Münzner equations. Moreover, it is easy to verify that $f=$ $\left.F\right|_{S^{2 l-1}}$ satisfies (cf. [GTY]):

$$
\left\{\begin{array}{l}
|\nabla f|^{2}=16\left(1-f^{2}\right), \\
\Delta f=8\left(m_{2}-m_{1}\right)-4(2 l+2) f,
\end{array}\right.
$$

where $m_{1}=m, m_{2}=l-m-1$. Thus by definition, $f$ is an isoparametric function on $S^{2 l-1}$.

According to [CCJ], [mm] and [Chi], all isoparametric hypersurfaces in spheres with four distinct principal curvatures are of FKM-type, except for the cases $\left(m_{1}, m_{2}\right)=$ $(2,2)$ and $(4,5)$, and except possibly for cases with multiplicities $(7,8)$, which has not been classified yet. As for the isoparametric hypersurfaces with $\left(m_{1}, m_{2}\right)=(2,2)$ or $(4,5)$, they must be homogeneous and thus unique ( $c f$. [OT], [Chi]). We are now ready to state the following

Theorem 1.2. For the focal submanifolds of an isoparametric hypersurface in $S^{n+1}$ with four distinct principal curvatures, we have

(i) All the $M_{-}$of FKM-type are not Einstein; the $M_{+}$of FKM-type is Einstein if and only if it is diffeomorphic to $S p(2)$ in the homogeneous case with $\left(m_{1}, m_{2}\right)=(4,3)$.

(ii) In the case $\left(m_{1}, m_{2}\right)=(2,2)$, the focal submanifold diffeomorphic to $\widetilde{G}_{2}\left(\mathbb{R}^{5}\right)$ is Einstein, while the other one diffeomorphic to $\mathbb{C} P^{3}$ is not.

(iii) In the case $\left(m_{1}, m_{2}\right)=(4,5)$, both focal submanifolds are not Einstein.

Remark 1.2. (1). In the FKM-family, there are two incongruent examples corresponding to $\left(m_{1}, m_{2}\right)=(4,3)$, one is homogeneous while the other is not. It is surprising that only the focal submanifold $M_{+}$of the homogenous case is Einstein. 
(2). For $\mathrm{g}=4$, we provide a complete determination for which focal submanifolds are Einstein except the case $\left(m_{1}, m_{2}\right)=(7,8)$, which has not been classified yet. Fortunately, we are able to show that for all the known examples with $\left(m_{1}, m_{2}\right)=(7,8)$ (in fact, three examples of FKM-type), the focal submanifolds are not Einstein.

\section{ISOPARAMETRIC FOLIATIONS}

2.1. Preliminaries. Let $M^{n}$ be an isoparametric hypersurface with four distinct principal curvatures in the unit sphere $S^{n+1}$, and $F$ be the corresponding Cartan-Münzner polynomial. In the current discussion, we focus only on the focal submanifold $M_{+}=$ $F^{-1}(1) \cap S^{n+1}$, since we can change $F$ to $-F$ so if necessary.

In virtue of Münzner, $M^{n}$ can be regarded as a unit normal sphere bundle $U N_{+}$ over $M_{+}$. In addition, at any point $x \in M_{+}$, the principal curvatures of the shape operator with respect to any unit normal vector are $0,1,-1$, with the corresponding multiplicities $m_{1}, m_{2}$ and $m_{2}$.

Following [CCJ], let $\left(x, n_{0}\right) \in U N_{+}$be points in a small open set, where $x \in M_{+}$ and $n_{0}$ is a unit normal vector of $M_{+}$at $x$. Adopting the following index ranges

$$
\begin{aligned}
& i, j, k \in\left\{1, \cdots, n=2\left(m_{1}+m_{2}\right)\right\}, \quad a, b, c \in\left\{1, \cdots, m_{1}\right\}, \\
& p, q, r \in\left\{m_{1}+1, \cdots, 2 m_{1}\right\}, \quad \alpha, \beta, \gamma \in\left\{2 m_{1}+1, \cdots, 2 m_{1}+m_{2}\right\}, \\
& \mu, \nu, \sigma \in\left\{2 m_{1}+m_{2}+1, \cdots, 2 m_{1}+2 m_{2}\right\}
\end{aligned}
$$

we choose a smooth orthonormal frame $\left\{n_{a}\right\},\left\{e_{p}\right\},\left\{e_{\alpha}\right\},\left\{e_{\mu}\right\}$ of $U N_{+}$in such a way that $\left\{n_{a}\right\}$ are tangent to the unit normal sphere at $n_{0}$, and $\left\{e_{p}\right\},\left\{e_{\alpha}\right\},\left\{e_{\mu}\right\}$ are respectively the basis vectors of the eigenspaces $V_{0}, V_{+}, V_{-}$of the shape operator $S_{n_{0}}$.

Since each frame vector can be regarded as a smooth map from $U N_{+}$to $\mathbb{R}^{n+2}$, using the Einstein summation convention, we have

$$
\begin{aligned}
& d x=\omega^{p} e_{p}+\omega^{\alpha} e_{\alpha}+\omega^{\mu} e_{\mu}, \\
& d n_{0}=\omega^{b} n_{b}-\omega^{\alpha} e_{\alpha}+\omega^{\mu} e_{\mu}, \\
& d n_{a}=-\omega^{a} n_{0}+\theta_{a}^{b} n_{b}+\theta_{a}^{q} e_{q}+\theta_{a}^{\alpha} e_{\alpha}+\theta_{a}^{\mu} e_{\mu}, \\
& d e_{p}=-\omega^{p} x+\theta_{p}^{b} n_{b}+\theta_{p}^{q} e_{q}+\theta_{p}^{\alpha} e_{\alpha}+\theta_{p}^{\mu} e_{\mu}, \\
& d e_{\alpha}=-\omega^{\alpha} x+\omega^{\alpha} n_{0}+\theta_{\alpha}^{b} n_{b}+\theta_{\alpha}^{q} e_{q}+\theta_{\alpha}^{\beta} e_{\beta}+\theta_{\alpha}^{\mu} e_{\mu}, \\
& d e_{\mu}=-\omega^{\mu} x-\omega^{\mu} n_{0}+\theta_{\mu}^{b} n_{b}+\theta_{\mu}^{q} e_{q}+\theta_{\mu}^{\alpha} e_{\alpha}+\theta_{\mu}^{\nu} e_{\nu},
\end{aligned}
$$


where

$$
\begin{aligned}
& \theta_{a}^{p}=\sum_{\alpha} F_{p a}^{\alpha} \omega^{\alpha}-\sum_{\mu} F_{p a}^{\mu} \omega^{\mu}, \theta_{a}^{\alpha}=\sum_{p} F_{p a}^{\alpha} \omega^{p}-2 \sum_{\mu} F_{\alpha a}^{\mu} \omega^{\mu} \\
& \theta_{p}^{\alpha}=\sum_{a} F_{p a}^{\alpha} \omega^{a}-2 \sum_{\mu} F_{\alpha p}^{\mu} \omega^{\mu}, \theta_{a}^{\mu}=-\sum_{p} F_{p a}^{\mu} \omega^{p}-2 \sum_{\alpha} F_{\alpha a}^{\mu} \omega^{\alpha}, \\
& \theta_{p}^{\mu}=\sum_{a} F_{p a}^{\mu} \omega^{a}+2 \sum_{\alpha} F_{\alpha p}^{\mu} \omega^{\alpha}, \theta_{\alpha}^{\mu}=\sum_{a} F_{\alpha a}^{\mu} \omega^{a}+\sum_{p} F_{\alpha p}^{\mu} \omega^{p} .
\end{aligned}
$$

Combining the third equation in (6) with (7), we obtain an explicit expression of $S_{a}:=S_{n_{a}}$ in terms of $F_{\alpha a}^{\mu}, F_{\alpha p}^{\mu}, F_{p a}^{\mu}, F_{p a}^{\alpha}$ defined above and the orthonormal coframe field $\omega^{p}, \omega^{\alpha}, \omega^{\mu}$ :

$$
S_{a}=\left(2 F_{\alpha a}^{\mu} e_{\mu}-F_{p a}^{\alpha} e_{p}\right) \omega^{\alpha}+\left(2 F_{\alpha a}^{\mu} e_{\alpha}+F_{p a}^{\mu} e_{p}\right) \omega^{\mu}+\left(-F_{p a}^{\alpha} e_{\alpha}+F_{p a}^{\mu} e_{\mu}\right) \omega^{p},
$$

Define linear operators ( [CCJ $]$ )

$$
\begin{aligned}
& A_{a}=2 F_{\alpha a}^{\mu} e_{\alpha} \omega^{\mu}: V_{-} \rightarrow V_{+}, \\
& B_{a}=-F_{p a}^{\alpha} e_{\alpha} \omega^{p}: V_{0} \rightarrow V_{+}, \\
& C_{a}=F_{p a}^{\mu} e_{\mu} \omega^{p}: \quad V_{0} \rightarrow V_{-},
\end{aligned}
$$

and their transposes

$$
\begin{aligned}
& { }^{t} A_{a}=2 F_{\alpha a}^{\mu} e_{\mu} \omega^{\alpha}: V_{+} \rightarrow V_{-}, \\
& { }^{t} B_{a}=-F_{p a}^{\alpha} e_{p} \omega^{\alpha}: V_{+} \rightarrow V_{0}, \\
& { }^{t} C_{a}=F_{p a}^{\mu} e_{p} \omega^{\mu}: \quad V_{-} \rightarrow V_{0} .
\end{aligned}
$$

With respect to the orthogonal direct sum decomposition

$$
T_{x} M_{+}=V_{+} \oplus V_{-} \oplus V_{0}=\operatorname{Span}\left\{e_{\alpha}\right\} \oplus \operatorname{Span}\left\{e_{\mu}\right\} \oplus \operatorname{Span}\left\{e_{p}\right\},
$$

the shape operator $S_{a}$ has the block form

$$
S_{a}=\left(\begin{array}{ccc}
0 & A_{a} & B_{a} \\
{ }^{t} A_{a} & 0 & C_{a} \\
{ }^{t} B_{a} & { }^{t} C_{a} & 0
\end{array}\right) .
$$

2.2. Proof of Theorem 1.1. Let $M^{n}$ be an isoparametric hypersurface with four distinct principal curvatures in the unit sphere $S^{n+1}$, and let $F$ be its Cartan-Münzner polynomial. We only make the proof for $M_{+}$, as the proof for $M_{-}$is analogous.

Take the same notations as in Section 2.1. Based on the principal decomposition $V_{+}, V_{0}$ and $V_{-}$of the tangent space $T M_{+}$, we simplify the criterion (11) for Willmore submanifolds to be:

$$
\sum_{\alpha} \operatorname{Ric}\left(e_{\alpha}\right)=\sum_{\mu} \operatorname{Ric}\left(e_{\mu}\right)
$$


At first, from Gauss equation, we derive that

$$
\begin{aligned}
\operatorname{Ric}\left(e_{\alpha}\right) & =\sum_{t=m_{1}+1}^{2 m_{1}+2 m_{2}} \bar{R}\left(e_{t}, e_{\alpha}, e_{t}, e_{\alpha}\right)+\sum_{t=m_{1}+1}^{2 m_{1}+2 m_{2}}\left\langle h\left(e_{t}, e_{t}\right), h\left(e_{\alpha}, e_{\alpha}\right)\right\rangle-\sum_{t=m_{1}+1}^{2 m_{1}+2 m_{2}}\left|h\left(e_{t}, e_{\alpha}\right)\right|^{2} \\
& =\left(m_{1}+2 m_{2}-1\right)+\sum_{t=m_{1}+1}^{2 m_{1}+2 m_{2}}\left\langle h\left(e_{t}, e_{t}\right), h\left(e_{\alpha}, e_{\alpha}\right)\right\rangle-\sum_{t=m_{1}+1}^{2 m_{1}+2 m_{2}}\left|h\left(e_{t}, e_{\alpha}\right)\right|^{2}
\end{aligned}
$$

where $\bar{R}$ is the curvature tensor of $S^{n+1}$.

Immediately, the minimality of $M_{+}$gives rise to

$$
\sum_{t}\left\langle h\left(e_{t}, e_{t}\right), h\left(e_{\alpha}, e_{\alpha}\right)\right\rangle=0 .
$$

Additionally, another straightforward calculation depending on the formula (8) leads us to the last item in the expression of $\operatorname{Ric}\left(e_{\alpha}\right)$ as follows

$$
\begin{aligned}
\sum_{t}\left|h\left(e_{t}, e_{\alpha}\right)\right|^{2} & =\sum_{t}\left|\sum_{a}\left\langle h\left(e_{t}, e_{\alpha}\right), n_{a}\right\rangle n_{a}+\left\langle h\left(e_{t}, e_{\alpha}\right), n_{0}\right\rangle n_{0}\right|^{2} \\
& =\sum_{a, t}\left\langle S_{a} e_{\alpha}, e_{t}\right\rangle^{2}+\sum_{t}\left\langle S_{0} e_{\alpha}, e_{t}\right\rangle^{2} \\
& =\sum_{a}\left|S_{a} e_{\alpha}\right|^{2}+1 \\
& =4 \sum_{a, \mu}\left(F_{\alpha a}^{\mu}\right)^{2}+\sum_{a, p}\left(F_{p a}^{\alpha}\right)^{2}+1 .
\end{aligned}
$$

In summary, we arrive at

$$
\sum_{\alpha} \operatorname{Ric}\left(e_{\alpha}\right)=m_{2}\left(m_{1}+2 m_{2}-2\right)-4 \sum_{a, \alpha, \mu}\left(F_{\alpha a}^{\mu}\right)^{2}-\sum_{a, p, \alpha}\left(F_{p a}^{\alpha}\right)^{2} .
$$

Similarly,

$$
\sum_{\mu} \operatorname{Ric}\left(e_{\mu}\right)=m_{2}\left(m_{1}+2 m_{2}-2\right)-4 \sum_{a, \alpha, \mu}\left(F_{\alpha a}^{\mu}\right)^{2}-\sum_{a, p, \mu}\left(F_{p a}^{\mu}\right)^{2} .
$$

Therefore, the equality $\sum_{\alpha} \operatorname{Ric}\left(e_{\alpha}\right)=\sum_{\mu} \operatorname{Ric}\left(e_{\mu}\right)$ holds if and only if

$$
\sum_{a, p, \alpha}\left(F_{p a}^{\alpha}\right)^{2}=\sum_{a, p, \mu}\left(F_{p a}^{\mu}\right)^{2}
$$

As claimed in Lemma 49 of [CCJ], for any choice of $a \in\left\{1, \ldots, m_{1}\right\}$, there is always an orthonormal basis in $V_{+}$and an orthonormal basis in $V_{-}$such that relative to these bases, $B_{a}=C_{a}$. In other words, by the definition (9) of $B_{a}$ and $C_{a}$, the equality (11) holds.

Now, the proof of Theorem 1.1 is complete. 


\section{FKM-TYPE ISOPARAMETRIC POLYNOMIALS}

In this section, we give a detailed study on the focal submanifolds of FKM-type and show a complete determination for which focal submanifolds are Einstein in this situation, providing a proof of 1) in Theorem 1.2 .

3.1. $M_{-}$of FKM-type. Recalling the Cartan-Münzner polynomial constructed by Ferus, Karcher and Münzner ([FKM] $)$ :

$$
F(x)=|x|^{4}-2 \sum_{i=0}^{m}\left\langle P_{i} x, x\right\rangle^{2},
$$

we find that

$$
\begin{aligned}
M_{-} & =F^{-1}(-1) \cap S^{2 l-1} \\
& =\left\{x \in S^{2 l-1} \mid \text { there exists } P \in \Sigma\left(P_{0}, \cdots, P_{m}\right) \text { with } P x=x\right\},
\end{aligned}
$$

where $\Sigma\left(P_{0}, \cdots, P_{m}\right)$ is the unit sphere in $\operatorname{Span}\left\{P_{0}, \cdots, P_{m}\right\}$, which is called the Clifford sphere. Notice that for any $P \in \Sigma\left(P_{0}, \cdots, P_{m}\right)$, we have $P^{2}=I$ and Trace $P=0$. Thus the eigenvalues of $P$ must be \pm 1 , with the same multiplicities. Denoting the corresponding eigenspaces by $E_{+}(P)$ and $E_{-}(P)$ respectively, we can decompose $\mathbb{R}^{2 l}$ as

$$
\mathbb{R}^{2 l}=E_{+}(P) \oplus E_{-}(P) .
$$

Let $y \in M_{-}$and $P \in \Sigma\left(P_{0}, \cdots, P_{m}\right)$ with $P y=y$. Define

$$
\Sigma_{P}:=\left\{Q \in \Sigma\left(P_{0}, \cdots, P_{m}\right) \mid\langle P, Q\rangle:=\frac{1}{2 l} \operatorname{Trace}(P Q)=0\right\},
$$

which is the equatorial sphere of $\Sigma\left(P_{0}, \cdots, P_{m}\right)$ orthogonal to $P$. In this way, we have a decomposition of the tangent space $T M_{-}$with respect to the eigenspaces of the shape operator.

Lemma ([FKM] $)$ The principal curvatures of the shape operator $S_{N}$ with respect to any unit normal vector $N \in T_{y}^{\perp} M_{-}$are 0,1 , and -1 , with the corresponding eigenspaces $\operatorname{Ker}\left(S_{N}\right), E_{+}\left(S_{N}\right), E_{-}\left(S_{N}\right)$ as follows:

$$
\begin{aligned}
\operatorname{Ker}\left(S_{N}\right) & =\left\{v \in E_{+}(P) \mid v \perp y, v \perp \Sigma_{P} N\right\}, \\
E_{+}\left(S_{N}\right) & =\mathbb{R} \Sigma_{P}(y+N), \\
E_{-}\left(S_{N}\right) & =\mathbb{R} \Sigma_{P}(y-N) .
\end{aligned}
$$

Moreover,

$$
\operatorname{dim} \operatorname{Ker}\left(S_{N}\right)=l-m-1, \operatorname{dim} E_{+}\left(S_{N}\right)=\operatorname{dim} E_{-}\left(S_{N}\right)=m .
$$


To facilitate the description, in front of the proof of 1) in Theorem 1.2, we state the following lemma, which is a direct corollary of the Gauss equation for the minimal submanifold.

Lemma 3.1. Let $M^{n}$ be an n-dimensional submanifold minimally immersed in an $(n+p)$-dimensional unit sphere $S^{n+p}$. Then $M$ is Einstein if and only if for arbitrary $x \in M$ and orthonormal basis $\left\{N_{\alpha}\right\}$ of $T_{x}^{\perp} M$,

$$
\sum_{\alpha=n+1}^{n+p}\left|S_{N_{\alpha}}(X)\right|^{2} \text { is constant, } \forall X \in T_{x} M \text { with }|X|=1,
$$

where $S_{N_{\alpha}}$ is the shape operator with respect to the normal vector $N_{\alpha}$.

With all these preparations, we are in a position to prove that the focal submanifold $M_{-}$of FKM-type is not Einstein.

Proof of 1) in Theorem 1.2 for $M_{-}$: Given $y \in M_{-}$and $P \in \Sigma\left(P_{0}, \cdots, P_{m}\right)$ with $P y=y$, let $\left\{N_{\alpha}\right\}_{\alpha=1}^{l-m}$ be an orthonormal basis for $T_{y}^{\perp} M_{-}$. For any $X \in T_{y} M_{-}$ with $|X|=1$, there is a decomposition

$$
X=X_{\alpha}^{0}+X_{\alpha}^{+}+X_{\alpha}^{-} \in \operatorname{Ker}\left(S_{N_{\alpha}}\right) \oplus E_{+}\left(S_{N_{\alpha}}\right) \oplus E_{-}\left(S_{N_{\alpha}}\right)
$$

with respect to $N_{\alpha}$ by the lemma of [FKM] mentioned before. For any $Q \in \Sigma_{P}$, we define $Y=Q y$, and decompose it as

$$
Y=\frac{1}{2} Q\left(y+N_{\alpha}\right)+\frac{1}{2} Q\left(y-N_{\alpha}\right) \in E_{+}\left(S_{N_{\alpha}}\right) \oplus E_{-}\left(S_{N_{\alpha}}\right)
$$

for any $\alpha$. Thus $Y \perp \cup_{N_{\alpha} \in T_{y}^{\perp} M_{-}} \operatorname{Ker}\left(S_{N_{\alpha}}\right)$, which implies immediately that

$$
\sum_{\alpha=1}^{l-m}\left|S_{N_{\alpha}}(Y)\right|^{2}=l-m-\sum_{\alpha=1}^{l-m}\left|Y_{\alpha}^{0}\right|^{2}=l-m .
$$

On the other hand, choosing $Z \in \operatorname{Ker}\left(S_{N_{1}}\right)$ with $|Z|=1$, we have

$$
\sum_{\alpha=1}^{l-m}\left|S_{N_{\alpha}}(Z)\right|^{2}=l-m-\sum_{\alpha=1}^{l-m}\left|Z_{\alpha}^{0}\right|^{2} \leq l-m-1 .
$$

Therefore, $M_{-}$is not Einstein by Lemma 3.1 ,

3.2. $M_{+}$of FKM-type. This subsection will be committed to proving 1) of Theorem 1.2 for the focal submanifold $M_{+}$of FKM-type.

We start with a description of the normal space of the focal submanifold

$$
M_{+}=\left\{x \in S^{2 l-1} \mid\left\langle P_{0} x, x\right\rangle=\ldots=\left\langle P_{m} x, x\right\rangle=0\right\} .
$$

As pointed out by [FKM], the normal space at $x \in M_{+}$is

$$
T_{x}^{\perp} M_{+}=\left\{P x \mid P \in \mathbb{R} \Sigma\left(P_{0}, \ldots, P_{m}\right)\right\} .
$$


Following [TY], $\forall X \in T_{x} M_{+}$with $|X|=1$, let $\left\{X=e_{1}, e_{2}, \ldots, e_{2 l-m-2}\right\}$ be an orthonormal basis for $T_{x} M_{+}$, and $\left\{P_{0} x, \ldots, P_{m} x\right\}$ an orthonormal basis for $T_{x}^{\perp} M_{+}$. Then from the Gauss equation and properties of Clifford system $\left\{P_{0}, \cdots, P_{m}\right\}$, we derive the Ricci curvature of $X$ :

$$
\operatorname{Ric}(X)=2(l-m-2)+2 \sum_{i, j=0, i<j}^{m}\left\langle X, P_{i} P_{j} x\right\rangle^{2} .
$$

As established by [TY in their concluding remark, a sufficient condition for $M_{+}$not to be Einstein can be stated as

$$
\operatorname{dim} M_{+}>\operatorname{dim} \operatorname{Span}\left\{P_{i} P_{j} x \mid i, j=0,1, \ldots, m, i<j\right\} .
$$

Moreover, [TY] reveals that the focal submanifold $M_{+}$of FKM-type is not Einstein, except possibly for those with multiplicities in one of the following pairs

$$
\left(m_{1}, m_{2}\right)=(m, l-m-1)=(4,3),(5,2),(6,1),(7,8),(8,7),(9,6),(10,21) .
$$

As asserted by [FKM], the isoparametric families of FKM-type with multiplicities $(5,2),(6,1)$ are congruent to those with multiplicities $(2,5),(1,6)$. Hence, both $M_{+}$ in the cases $\left(m_{1}, m_{2}\right)=(5,2)$, and $(6,1)$ are not Einstein by the proved result for $M_{-}$ in last subsection.

Consequently, the cases with $\left(m_{1}, m_{2}\right)=(4,3),(7,8),(8,7),(9,6),(10,21)$ are left to our consideration. Since the $(4,3)$ case is the most amazing one, we will firstly deal with this case.

3.2.1. The (4,3) case. In this case, $m=4$ and $l=8=2 \delta(4)$, where $\delta(m)$ is the dimension of irreducible representation of $C_{m-1}$. According to [FKM], there are two examples of FKM-type isoparametric polynomials with multiplicities $(4,3)$, which are distinguished by an invariant

$$
\operatorname{Trace}\left(P_{0} P_{1} P_{2} P_{3} P_{4}\right)=2 q \delta(4), \text { with } q \equiv 2 \bmod 2 .
$$

Noticing that $P_{0} P_{1} P_{2} P_{3} P_{4}$ is a symmetric orthogonal matrix on $\mathbb{R}^{16}$, we divide the proof into two parts.

1). $q=2$. On this condition, $P_{0} P_{1} P_{2} P_{3} P_{4}$ is a symmetric orthogonal matrix on $\mathbb{R}^{16}$ with Trace $\left(P_{0} P_{1} P_{2} P_{3} P_{4}\right)=16$. Thus it follows easily that $P_{0} P_{1} P_{2} P_{3} P_{4}=I_{16}$, which makes the elements in $\left\{P_{i} P_{j} x \mid i, j=0,1, \ldots, 4, i<j\right\}$ perpendicular to each other. For example, we have

$$
\begin{aligned}
& \left\langle P_{0} P_{1} x, P_{0} P_{2} x\right\rangle=\left\langle P_{1} x, P_{2} x\right\rangle=\left\langle P_{1}, P_{2}\right\rangle\langle x, x\rangle=0 \\
& \left\langle P_{1} P_{2} x, P_{3} P_{4} x\right\rangle=\left\langle P_{1} P_{2} x, P_{0} P_{1} P_{2} P_{3} P_{4} P_{3} P_{4} x\right\rangle=-\left\langle P_{0} x, x\right\rangle=0 .
\end{aligned}
$$


As indicated in [TY], $\operatorname{Span}\left\{P_{i} P_{j} x \mid i, j=0,1, \ldots, 4, i<j\right\} \subset T_{x} M_{+}$. Observing that $\#\left\{P_{i} P_{j} x \mid i, j=0,1, \ldots, 4, i<j\right\}=\operatorname{dim} T_{x} M_{+}=10$, we find that $\left\{P_{i} P_{j} x \mid i, j=\right.$ $0,1, \ldots, 4, i<j\}$ constitutes an orthonormal basis of $T_{x} M_{+}$.

Hence, based on the formula (14), a fundamental argument in linear algebra shows immediately that $M_{+}$corresponding to $q=2$ is Einstein!

According to [FKM], this example corresponding to $q=2$ is the homogeneous one. By the classification of homogeneous hypersurfaces in spheres ( $c f$. [TT] $), M_{+}$in this case is diffeomorphic to $S p(2)$.

2). $q=0$. On this condition, we have $\operatorname{Trace}\left(P_{0} P_{1} P_{2} P_{3} P_{4}\right)=0$. Then there exists $T \in O(16)$, such that $P_{0} P_{1} P_{2} P_{3} P_{4}=T^{t}\left(\begin{array}{cc}I & 0 \\ 0 & -I\end{array}\right) T$. Suppose $M_{+}$is Einstein in this case. Then it is obvious that $\left\{P_{i} P_{j} x \mid i, j=0,1, \ldots, 4, i<j\right\}$ forms an orthonormal basis of $T_{x} M_{+}$for any $x \in M_{+}$. Hence, a simple verification leads to

$$
\begin{aligned}
& \left\langle P_{0} P_{1} P_{2} P_{3} x, x\right\rangle=-\left\langle P_{0} P_{1} x, P_{2} P_{3} x\right\rangle=0, \\
& \left\langle P_{0} P_{1} P_{2} P_{3} x, P_{i} P_{j} x\right\rangle=0, \text { for } 0 \leq i<j \leq 4,
\end{aligned}
$$

which imply that $P_{0} P_{1} P_{2} P_{3} x \in T_{x}^{\perp} M_{+}$. Moreover, from the identities

$$
\left\langle P_{0} x, P_{0} P_{1} P_{2} P_{3} x\right\rangle=\left\langle P_{1} x, P_{0} P_{1} P_{2} P_{3} x\right\rangle=\left\langle P_{2} x, P_{0} P_{1} P_{2} P_{3} x\right\rangle=\left\langle P_{3} x, P_{0} P_{1} P_{2} P_{3} x\right\rangle=0,
$$

it follows that $P_{0} P_{1} P_{2} P_{3} x= \pm P_{4} x$. That is to say,

$$
T^{t}\left(\begin{array}{cc}
I & 0 \\
0 & -I
\end{array}\right) T x= \pm x .
$$

Write $T x=\left(y^{t}, z^{t}\right)^{t}$. Substituting into (16), it follows directly that $y=0$ or $z=0$. In other words, we get a map $T: M_{+}^{10} \rightarrow S^{15}$ mapping $x$ to $T x$ with $T\left(M_{+}^{10}\right) \subset$ $S^{7}(1) \times\{0\} \cup\{0\} \times S^{7}(1)$, which contradicts the fact that $T$ is an orthogonal matrix.

In conclusion, $M_{+}$is not Einstein in this case!

Remark 3.1. According to [FKM], this example corresponding to $q=0$ is the inhomogeneous one, which is congruent to the unique example of FKM-type with multiplicities $(3,4)$. Thus $M_{+}$of this example is isometric to $M_{-}$of FKM-type with multiplicities $(3,4)$, which is not Einstein by the proved result in Section 3.1.

3.2.2. The $(7,8)$ case. The key point for the proof in this case is an interesting lemma as we state below, which relates to the condition (A) introduced by Ozeki and Takeuchi $\mathrm{OT}$. We remark that this condition (A) was interpreted as a condition on the second fundamental form by [FKM]. 
Lemma 3.2. Let $M$ be a submanifold minimally immersed in the unit sphere. If $M$ satisfies condition (A), i.e. at some point of $M$, the kernels of all shape operators $S_{N}(N \neq 0)$ coincide, then $M$ is not Einstein.

Proof. It is an immediate corollary of Lemma 3.1

From Theorem 5.8 in [FKM], we see that $M_{+}$in the $(7,8)$ case does satisfy the condition (A). Consequently, $M_{+}$is not Einstein.

3.2.3. The $(\mathbf{9}, \boldsymbol{6})$ case. In this case, $m=9$ and $l=16$. For the Clifford system $\left\{P_{0}, \cdots, P_{9}\right\}$ on $\mathbb{R}^{32}$, we choose $x \in S^{31}$ to be a common eigenvector of the commuting operators

$$
P_{2 i} P_{2 i+1} P_{2 j} P_{2 j+1}, 0 \leq i<j \leq 4 .
$$

Observing that each $P_{i}$ anti-commutes with at least one of these operators, we see $x \in M_{+}$. Furthermore, since $x$ is also an eigenvector of the product of the operators mentioned above, we obtain the following identities:

$$
\begin{aligned}
& P_{0} P_{1} x= \pm P_{2} P_{3} x= \pm P_{4} P_{5} x= \pm P_{6} P_{7} x= \pm P_{8} P_{9} x, \\
& P_{0} P_{2} x= \pm P_{1} P_{3} x, P_{0} P_{3} x= \pm P_{1} P_{2} x, \\
& P_{0} P_{4} x= \pm P_{1} P_{5} x, P_{0} P_{5} x= \pm P_{1} P_{4} x, \\
& P_{0} P_{6} x= \pm P_{1} P_{7} x, P_{0} P_{7} x= \pm P_{1} P_{6} x, \\
& P_{0} P_{8} x= \pm P_{1} P_{9} x, P_{0} P_{9} x= \pm P_{1} P_{8} x, \\
& P_{2} P_{4} x= \pm P_{3} P_{5} x, P_{2} P_{5} x= \pm P_{3} P_{4} x, \\
& P_{2} P_{6} x= \pm P_{3} P_{7} x, P_{2} P_{7} x= \pm P_{3} P_{6} x, \\
& P_{2} P_{8} x= \pm P_{3} P_{9} x, P_{2} P_{9} x= \pm P_{3} P_{8} x, \\
& P_{4} P_{6} x= \pm P_{5} P_{7} x, P_{4} P_{7} x= \pm P_{5} P_{6} x, \\
& P_{4} P_{8} x= \pm P_{5} P_{9} x, P_{4} P_{9} x= \pm P_{5} P_{8} x, \\
& P_{6} P_{8} x= \pm P_{7} P_{9} x, P_{6} P_{9} x= \pm P_{7} P_{8} x .
\end{aligned}
$$

As a direct result, $\operatorname{dim} \operatorname{Span}\left\{P_{i} P_{j} x \mid i, j=0,1, \ldots, 9, i<j\right\} \leq 21=\operatorname{dim} T_{x} M_{+}$. Moreover, from the formula (14) of Ricci curvature of $M_{+}$, for any $X \in T_{x} M_{+}$, we derive that

$$
\begin{aligned}
\operatorname{Ric}(X)= & 10+2\left\{5\left\langle X, P_{0} P_{1} x\right\rangle^{2}+2 \sum_{i=2}^{9}\left\langle X, P_{0} P_{i} x\right\rangle^{2}+2 \sum_{i=4}^{9}\left\langle X, P_{2} P_{i} x\right\rangle^{2}\right. \\
& \left.+2 \sum_{i=6}^{9}\left\langle X, P_{4} P_{i} x\right\rangle^{2}+2 \sum_{i=8}^{9}\left\langle X, P_{6} P_{i} x\right\rangle^{2}\right\}
\end{aligned}
$$

At last, by the following lemma in linear algebra, $M_{+}$is not Einstein in this case. 
Lemma 3.3. Let $\left\{u_{1}, \cdots, u_{21}\right\}$ be a class of unit vectors in $\mathbb{R}^{21}$. Then $\rho(X):=$ $5\left\langle X, u_{1}\right\rangle^{2}+2 \sum_{i=2}^{21}\left\langle X, u_{i}\right\rangle^{2}$ is not constant on the unit sphere in $\mathbb{R}^{21}$.

3.2.4. The $(\boldsymbol{8}, \boldsymbol{7})$ case. In this case, $m=8$ and $l=16$. By the representation theory of Clifford algebra, we can extend a Clifford system $\left\{P_{0}, \cdots, P_{8}\right\}$ on $\mathbb{R}^{32}$ to a system $\left\{P_{0}, \cdots, P_{9}\right\}$. Let $x \in S^{31}$ again be a common eigenvector of the commuting operators

$$
P_{2 i} P_{2 i+1} P_{2 j} P_{2 j+1}, 0 \leq i<j \leq 4 .
$$

Repeating the arguments in 3.2 .3 , we see that $x$ belongs to $M_{+}$and

$$
\operatorname{dim} \operatorname{Span}\left\{P_{i} P_{j} x \mid i, j=0,1, \ldots, 9, i<j\right\} \leq 21<22=\operatorname{dim} T_{x} M_{+} .
$$

Again, using the formula (14) for the Ricci curvature, we conclude that $M_{+}$is not Einstein in this case.

In fact, there are two incongruent families of FKM-type with multiplicities $(8,7)$, neither is congruent to the $(7,8)$ family, and both $M_{+}$of the two families are not Einstein.

3.2.5. The $(10,21)$ case. In this case, $m=10$ and $l=32$. Choosing $x \in S^{63}$ to be a common eigenvector of the commuting operators

$$
P_{0} P_{1} P_{2} P_{3}, P_{4} P_{5} P_{6} P_{7}, P_{0} P_{1} P_{8} P_{9}, P_{2} P_{3} P_{8} P_{9}, P_{0} P_{2} P_{8} P_{10},
$$

we see that $x \in M_{+}$. Thus a similar argument as in 3.2.3 implies that

$$
\operatorname{dim} \operatorname{Span}\left\{P_{i} P_{j} x \mid i, j=0,1, \ldots, 10, i<j\right\}<52=\operatorname{dim} T_{x} M_{+} .
$$

Therefore, $M_{+}$is not Einstein in this case.

Up to now, the proof of 1 ) in Theorem 1.2 is complete!

\section{Homogeneous isoparametric hypersurfaces}

This section will be committed to investigating the homogeneous cases with multiplicities $(2,2)$ and $(4,5)$.

We begin by recalling a formulation of the Cartan-Münzner polynomial $F$ in terms of the second fundamental forms of the focal submanifolds, developed by Ozeki and Takeuchi in [OT] (see also [CCJ], p.52). For $x \in M_{+}$, and an orthonormal basis $\left\{n_{0}, n_{1}, \cdots, n_{m_{1}}\right\}$ of the normal space $T_{x}^{\perp} M_{+}$, we introduce the quadratic homogeneous polynomials

$$
p_{i}(y):=\left\langle S_{n_{i}} y, y\right\rangle, \quad 0 \leq i \leq m_{1}
$$


where $y \in T_{x} M_{+}$. The Cartan-Münzner polynomial F can be expressed by $p_{i}$ as follows,

$$
\begin{aligned}
F(t x+y+w)= & t^{4}+\left(2|y|^{2}-6|w|^{2}\right) t^{2}+8\left(\sum_{i=0}^{m_{1}} p_{i}(y) w_{i}\right) t \\
& +|y|^{4}-2 \sum_{i=0}^{m_{1}}\left(p_{i}(y)\right)^{2}+8 \sum_{i=0}^{m_{1}} q_{i}(y) w_{i} \\
& +2 \sum_{i, j=0}^{m_{1}}\left\langle\nabla p_{i}, \nabla p_{j}\right\rangle w_{i} w_{j}-6|y|^{2}|w|^{2}+|w|^{4}
\end{aligned}
$$

where the homogeneous polynomials of degree three, $q_{i}(y)$, are the components of the third fundamental form of $M_{+}, w=\sum_{i=0}^{m_{1}} w_{i} n_{i}$.

4.1. The homogeneous example of multiplicities $(\mathbf{2 , 2})$. Consider the lie algebra $s o(5, \mathbb{R})$. The special orthogonal group $S O(5)$ acts on it by the adjoint representation

$$
g \cdot Z=g Z g^{-1}
$$

for $g \in S O(5)$ and $Z \in \operatorname{so}(5, \mathbb{R})$. Then the principal orbits of this action constitute the homogeneous 1-parameter family of isoparametric hypersurfaces in $S^{9}$ with multiplicities $\left(m_{1}, m_{2}\right)=(2,2)$. Denote the $(i, j)$-entry of $Z$ by $a_{i j} \in \mathbb{R}$. Then the Euclidean space $\mathbb{R}^{10}$ is $s o(5, \mathbb{R})$ coordinated by $a_{i j}$ with $i<j$, and according to $[\mathrm{OT}$, the CartanMünzner polynomial is

$$
\begin{aligned}
F(Z) & =\frac{3}{4}\left(\operatorname{Trace} Z^{2}\right)^{2}-2 \operatorname{Trace}\left(Z^{4}\right) \\
& =-\frac{5}{4} \sum_{i}\left|Z_{i}\right|^{4}+\frac{3}{2} \sum_{i<j}\left|Z_{i}\right|^{2}\left|Z_{j}\right|^{2}-4 \sum_{i<j}\left\langle Z_{i}, Z_{j}\right\rangle^{2},
\end{aligned}
$$

where $Z_{i}=\left(a_{i 1}, \cdots, a_{i 5}\right)$ is the row vector of $Z=\left(a_{i j}\right)$.

Making use of the expansion formula (18), we will calculate the second fundamental forms for the focal submanifolds $M_{+}:=F^{-1}(1) \cap S^{9}$ and $M_{-}:=F^{-1}(-1) \cap S^{9}$ at some special points, respectively.

1). The geometry of $M_{-}$.

Let $e$ be the point in $s o(5, \mathbb{R})$ coordinated by

$$
\left\{\begin{array}{l}
a_{12}=-a_{21}=1, \\
a_{i j}=0, \text { otherwise. }
\end{array}\right.
$$

Substituting $e$ into (19), we get immediately that $F(e)=-1$. It is easy to see that the isotropy subgroup at $e$ is $S O(2) \times S O(3)$, thus $M_{-}$is diffeomorphic to $\widetilde{G}_{2}\left(\mathbb{R}^{5}\right)=$ $\frac{S O(5)}{S O(2) \times S O(3)}$. 
Taking $e$ as the reference point, we can expand the polynomial $-F$ with respect to $a_{12}$ :

$$
\begin{aligned}
-F= & a_{12}^{4}+a_{12}^{2}\left\{2\left(a_{13}^{2}+a_{14}^{2}+a_{15}^{2}+a_{23}^{2}+a_{24}^{2}+a_{25}^{2}\right)-6\left(a_{34}^{2}+a_{35}^{2}+a_{45}^{2}\right)\right\} \\
& +16 a_{12}\left\{a_{34}\left(a_{24} a_{13}-a_{23} a_{14}\right)+a_{35}\left(a_{25} a_{13}-a_{23} a_{15}\right)+a_{45}\left(a_{25} a_{14}-a_{24} a_{15}\right)\right\} \\
& +G,
\end{aligned}
$$

where $G$ denotes the sum of other items containing no $a_{12}$. Comparing this expansion with (18), we find that $\left\{a_{34}, a_{35}, a_{45}\right\}$ and $\left\{a_{13}, a_{14}, a_{15}, a_{23}, a_{24}, a_{25}\right\}$ are respectively the normal and tangent coordinates. Setting

$$
w_{0}=a_{34}, w_{1}=a_{35}, w_{2}=a_{45},
$$

we have from (18) that

$$
\begin{aligned}
& p_{0}=2\left(a_{24} a_{13}-a_{23} a_{14}\right), \\
& p_{1}=2\left(a_{25} a_{13}-a_{23} a_{15}\right), \\
& p_{2}=2\left(a_{25} a_{14}-a_{24} a_{15}\right) .
\end{aligned}
$$

Furthermore, using (17), a direct calculation leads to

$$
\left|S_{0} X\right|^{2}+\left|S_{1} X\right|^{2}+\left|S_{2} X\right|^{2}=2\left(a_{13}^{2}+a_{14}^{2}+a_{15}^{2}+a_{23}^{2}+a_{24}^{2}+a_{25}^{2}\right),
$$

for any unit tangent vector $X=\left(a_{13}, a_{14}, a_{15}, a_{23}, a_{24}, a_{25}\right)$. From Lemma 3.1 and the homogeneity of $M_{-}$, it follows that $M_{-}$is Einstein.

Remark 4.1. Noticing that $M_{-}$is diffeomorphic to the Grassmann manifold $\widetilde{G}_{2}\left(\mathbb{R}^{5}\right)$ of the oriented two-planes in $\mathbb{R}^{5}$, it is natural to ask that if the induced metric on $M_{-}$ from the Euclidean space $\mathbb{R}^{10}$ is the unique invariant metric on the compact irreducible symmetric space $\widetilde{G}_{2}\left(\mathbb{R}^{5}\right)$. The answer is affirmative: in virtue of Lemma 1.8 in [Sol], $M_{-} \subset \mathbb{R}^{10}$ is just the standard Plücker embedding of $\widetilde{G}_{2}\left(\mathbb{R}^{5}\right)$ into $\mathbb{R}^{10}$.

2). The geometry of $M_{+}$.

Choose a point $e^{\prime}$ with coordinates $a_{12}=a_{34}=\frac{1}{\sqrt{2}}$ and zero otherwise. Clearly, $F\left(e^{\prime}\right)=1$. By a computation of the isotropy subgroup at $e^{\prime}$ (cf. [TXY]), we see that $M_{+}$is diffeomorphic to $\frac{S O(5)}{U(2)} \cong \mathbb{C} P^{3}$. In terms of new coordinates we introduced below

$$
\begin{aligned}
a_{12} & :=\left(t+w_{0}\right) / \sqrt{2}, a_{34}:=\left(t-w_{0}\right) / \sqrt{2}, \\
a_{13} & :=\left(w_{2}-z_{2}\right) / \sqrt{2}, a_{24}:=\left(w_{2}+z_{2}\right) / \sqrt{2}, \\
a_{14} & :=\left(z_{1}-w_{1}\right) / \sqrt{2}, a_{23}:=\left(z_{1}+w_{1}\right) / \sqrt{2},
\end{aligned}
$$

and

$$
x_{1}:=a_{35}, x_{2}:=a_{45}, y_{1}:=a_{15}, y_{2}:=x_{25},
$$


$F$ can be expanded with respect to $t$ as

$$
\begin{aligned}
F= & t^{4}+\left\{2\left(x_{1}^{2}+x_{2}^{2}+y_{1}^{2}+y_{2}^{2}+z_{1}^{2}+z_{2}^{2}\right)-6\left(w_{0}^{2}+w_{1}^{2}+w_{2}^{2}\right)\right\} t^{2} \\
& +8\left\{\left(x_{1}^{2}+x_{2}^{2}-y_{1}^{2}-y_{2}^{2}\right) w_{0}+2\left(x_{1} y_{1}+x_{2} y_{2}\right) w_{1}+2\left(x_{2} y_{1}-x_{1} y_{2}\right) w_{2}\right\} t+G^{\prime},
\end{aligned}
$$

where $G^{\prime}$ denotes the sum of other items containing no $t$. Comparing this expansion with (18), we find that $\left\{w_{0}, w_{1}, w_{2}\right\}$ and $\left\{x_{1}, x_{2}, y_{1}, y_{2}, z_{1}, z_{2}\right\}$ are respectively the normal and tangent coordinates. The components of the second fundamental form of $M_{+}$ at $e^{\prime}$ are

$$
\begin{aligned}
& p_{0}=x_{1}^{2}+x_{2}^{2}-y_{1}^{2}-y_{2}^{2}, \\
& p_{1}=2\left(x_{1} y_{1}+x_{2} y_{2}\right), \\
& p_{2}=2\left(x_{2} y_{1}-x_{1} y_{2}\right) .
\end{aligned}
$$

Therefore, for any $X=\left(x_{1}, x_{2}, y_{1}, y_{2}, z_{1}, z_{2}\right) \in T_{e^{\prime}} M_{+}$with $|X|=1$,

$$
\left|S_{0} X\right|^{2}+\left|S_{1} X\right|^{2}+\left|S_{2} X\right|^{2}=3\left(x_{1}^{2}+x_{2}^{2}+y_{1}^{2}+y_{2}^{2}\right),
$$

which is not constant, namely, $M_{+}$is not Einstein by Lemma 3.1 .

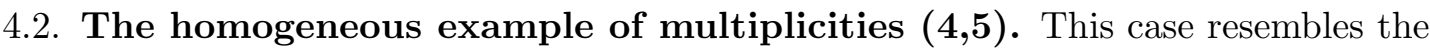
$(2,2)$ case strongly. However, both focal submanifolds $M_{+}$and $M_{-}$in this case are not Einstein. To show the assertion, consider the Lie algebra $s o(5, \mathbb{C})$. The unitary group $U(5)$ acts on it by the adjoint representation

$$
g \cdot Z=\bar{g} Z g^{-1}
$$

for $g \in U(5)$ and $Z \in s o(5, \mathbb{C})$. The principal orbits of this action constitute the homogeneous 1-parameter family of isoparametric hypersurfaces in $S^{19}$ with multiplicities $\left(m_{1}, m_{2}\right)=(4,5)$. Denote the $(i, j)$-entry of $Z$ by $a_{i j}=x_{i j}+\sqrt{-1} y_{i j}$ with real $x_{i j}$ and $y_{i j}$. Then the Euclidean space $\mathbb{R}^{20}$ is $s o(5, \mathbb{C})$ coordinated by $x_{i j}$ and $y_{i j}$ with $i<j$. Again, according to $\mathrm{OT}$, the Cartan-Münzner polynomial is

$$
\begin{aligned}
F(Z) & =\frac{3}{4}(\operatorname{Trace} Z \bar{Z})^{2}-2 \operatorname{Trace}(Z \bar{Z})^{2}, \\
& =-\frac{5}{4} \sum_{i}\left|Z_{i}\right|^{4}+\frac{3}{2} \sum_{i<j}\left|Z_{i}\right|^{2}\left|Z_{j}\right|^{2}-4 \sum_{i<j}\left|\left\langle Z_{i}, Z_{j}\right\rangle\right|^{2},
\end{aligned}
$$

where $Z_{i}=\left(a_{i 1}, \cdots, a_{i 5}\right)$ is the row vector of $Z=\left(a_{i j}\right)$ and $\left\langle Z_{i}, Z_{j}\right\rangle$ is the Hermitian inner product .

Comparing with the expansion formula (18), we will investigate the second fundamental forms for the focal submanifolds $M_{+}:=F^{-1}(1) \cap S^{19}$ and $M_{-}:=F^{-1}(-1) \cap S^{19}$ respectively.

1). Geometry of $M_{+}$. 
Following [Chi], we choose a point $e$ with coordinates $x_{12}=x_{34}=\frac{1}{\sqrt{2}}$ and zero otherwise. Clearly, $F(e)=1$. In a similar way as 2 ) in 4.1 , we introduce new coordinates

$$
\begin{aligned}
& x_{12}:=\left(t+w_{0}\right) / \sqrt{2}, x_{34}:=\left(t-w_{0}\right) / \sqrt{2}, \\
& x_{13}:=\left(w_{3}-z_{4}\right) / \sqrt{2}, x_{24}:=\left(w_{3}+z_{4}\right) / \sqrt{2}, \\
& y_{13}:=\left(-z_{3}-w_{4}\right) / \sqrt{2}, y_{24}:=\left(-z_{3}+w_{4}\right) / \sqrt{2}, \\
& x_{14}:=\left(z_{2}-w_{1}\right) / \sqrt{2}, x_{23}:=\left(z_{2}+w_{1}\right) / \sqrt{2}, \\
& y_{14}:=\left(w_{2}+z_{1}\right) / \sqrt{2}, y_{23}:=\left(w_{2}-z_{1}\right) / \sqrt{2},
\end{aligned}
$$

and

$$
\begin{aligned}
& x_{1}:=x_{35}, x_{2}:=y_{35}, x_{3}:=x_{45}, x_{4}:=y_{45}, x_{5}:=y_{34}, \\
& y_{1}:=x_{15}, y_{2}:=y_{15}, y_{3}:=x_{25}, y_{4}:=y_{25}, y_{5}:=y_{12} .
\end{aligned}
$$

Then $\left(w_{0}, w_{1}, w_{2}, w_{3}, w_{4}\right)$ are the normal coordinates, $\left(x_{1}, \ldots, x_{5}, y_{1}, \ldots, y_{5}, z_{1}, \ldots, z_{4}\right)$ are the tangent coordinates. Expanding $F$ with respect to $t$, the components of the second fundamental form of $M_{+}$at $e$ are given by

$$
\begin{aligned}
& p_{0}=x_{1}^{2}+\cdots+x_{5}^{2}-y_{1}^{2}-\cdots-y_{5}^{2}, \\
& p_{1}=2\left(x_{1} y_{1}+\cdots+x_{4} y_{4}\right)+\sqrt{2}\left(x_{5}+y_{5}\right) z_{1}, \\
& p_{2}=2\left(x_{2} y_{1}-x_{1} y_{2}\right)+2\left(x_{3} y_{4}-x_{4} y_{3}\right)+\sqrt{2}\left(x_{5}+y_{5}\right) z_{2}, \\
& p_{3}=2\left(x_{3} y_{1}-x_{1} y_{3}\right)+2\left(x_{4} y_{2}-x_{2} y_{4}\right)+\sqrt{2}\left(x_{5}+y_{5}\right) z_{3}, \\
& p_{4}=2\left(x_{2} y_{3}-x_{3} y_{2}\right)+2\left(x_{4} y_{1}-x_{1} y_{4}\right)+\sqrt{2}\left(x_{5}+y_{5}\right) z_{4} .
\end{aligned}
$$

Therefore, for any $X=\left(x_{1}, \cdots, x_{5}, 0, \cdots, 0,0, \cdots, 0\right) \in T_{e} M_{+}$,

$$
\sum_{i=0}^{4}\left|S_{i} X\right|^{2}=5 \sum_{\alpha=1}^{5} x_{\alpha}^{2}-2 x_{5}^{2},
$$

which implies that $M_{+}$is not Einstein by Lemma 3.1. We remark that $M_{+}$is diffeomorphic to the homogeneous space $\frac{U(5)}{S p(2) \times U(1)}\left(c f\right.$. [TXY]). In fact, it fibers over $\mathbb{C} P^{4}$ with fiber $\frac{U(4)}{S p(2)}$.

2). Geometry of $M_{-}$.

Let $e^{\prime}$ be a point with coordinates $x_{12}=-x_{21}=1$ and zero otherwise. Clearly, we have $F\left(e^{\prime}\right)=-1$. With respect to $x_{12},-F$ can be expanded as:

$$
-F=x_{12}^{4}+A x_{12}^{2}+8 B x_{12}+C
$$


where

$$
\begin{aligned}
A= & 2\left(y_{12}^{2}+\left|a_{13}\right|^{2}+\left|a_{14}\right|^{2}+\left|a_{15}\right|^{2}+\left|a_{23}\right|^{2}+\left|a_{24}\right|^{2}+\left|a_{25}\right|^{2}\right) \\
& -6\left(\left|a_{34}\right|^{2}+\left|a_{35}\right|^{2}+\left|a_{45}\right|^{2}\right), \\
B= & x_{34}\left(-2 x_{14} x_{23}+2 x_{13} x_{24}+2 y_{14} y_{23}-2 y_{13} y_{24}\right) \\
& +x_{35}\left(-2 x_{15} x_{23}+2 x_{13} x_{25}+2 y_{15} y_{23}-2 y_{13} y_{25}\right) \\
& +x_{45}\left(-2 x_{15} x_{24}+2 x_{14} x_{25}+2 y_{15} y_{24}-2 y_{14} y_{25}\right) \\
& +y_{34}\left(-2 x_{14} x_{23}+2 x_{13} y_{24}-2 y_{14} x_{23}+2 y_{13} x_{24}\right) \\
& +y_{35}\left(-2 x_{15} y_{23}+2 x_{13} y_{25}-2 y_{15} x_{23}+2 y_{13} x_{25}\right) \\
& +y_{45}\left(-2 x_{15} y_{24}+2 x_{14} y_{25}-2 y_{15} x_{24}+2 y_{14} x_{25}\right)
\end{aligned}
$$

$C$ denotes the sum of those items containing no $x_{12}$. From this expansion formula, we see that $\left\{x_{34}, x_{35}, x_{45}, y_{34}, y_{35}, y_{45}\right\}$ and $\left\{y_{12}, x_{13}, y_{13}, x_{14}, y_{14}, x_{15}, y_{15}, x_{23}\right.$, $\left.y_{23}, x_{24}, y_{24}, x_{25}, y_{25}\right\}$ are respectively the normal and tangent coordinates. Setting

$$
w_{0}=x_{34}, w_{1}=x_{35}, w_{2}=x_{45}, w_{3}=y_{34}, w_{4}=y_{35}, w_{5}=y_{45},
$$

we get

$$
\begin{aligned}
& p_{0}=-2 x_{14} x_{23}+2 x_{13} x_{24}+2 y_{14} y_{23}-2 y_{13} y_{24}, \\
& p_{1}=-2 x_{15} x_{23}+2 x_{13} x_{25}+2 y_{15} y_{23}-2 y_{13} y_{25}, \\
& p_{2}=-2 x_{15} x_{24}+2 x_{14} x_{25}+2 y_{15} y_{24}-2 y_{14} y_{25}, \\
& p_{3}=-2 x_{14} x_{23}+2 x_{13} y_{24}-2 y_{14} x_{23}+2 y_{13} x_{24}, \\
& p_{4}=-2 x_{15} y_{23}+2 x_{13} y_{25}-2 y_{15} x_{23}+2 y_{13} x_{25}, \\
& p_{5}=-2 x_{15} y_{24}+2 x_{14} y_{25}-2 y_{15} x_{24}+2 y_{14} x_{25} .
\end{aligned}
$$

Observing that the unit tangent vector $Y_{12}$ with $y_{12}=1$ is contained in $\operatorname{Ker}\left(S_{i}\right)$ for $0 \leq i \leq 5$, we derive from the formulas above that

$$
\sum_{i=0}^{5}\left|S_{i}\left(Y_{12}\right)\right|^{2}=0
$$

On the other hand, there does exist a unit tangent vector $v$ such that

$$
\sum_{i=0}^{5}\left|S_{i} v\right|^{2} \neq 0 .
$$

This leads us to the final conclusion that $M_{-}$in the $(4,5)$ case is not Einstein by Lemma 3.1. We remark that $M_{+}$is diffeomorphic to $\frac{U(5)}{S U(2) \times U(3)}(c f$. [TXY] $)$.

Acknowledgements . The authors would like to thank Professor Q.S.Chi for his excellent lectures on isoparametric hypersurfaces given at Beijing Normal University and valuable discussions. They also express their sincere gratitude to Professor T.E.Cecil 
for his handwritten translation of [FKM] and many helpful comments during the third author preparing the Latex version of the manuscript.

\section{REFERENCES}

[CCJ] Thomas E. Cecil, Quo-Shin Chi, and Gary R. Jensen, Isoparametric hypersurfaces with four principal curvatures, Ann. of Math. (2) 166 (2007), no. 1, 1-76.

[CR] T. E. Cecil and P. T. Ryan, Tight and taut immersions of manifolds, Research Notes in Math. 107, Pitman, London, 1985.

[Chi] Quo-Shin Chi, Isoparametric hypersurfaces with four principal curvatures, II and III, Nagoya Math. J. 204 (2011), 1-18, and preprint, 2011, arXiv:1104.3249.

[FKM] D. Ferus, H. Karcher, and H. F. Münzner, Cliffordalgebren und neue isoparametrische Hyperflächen, Math. Z. 177 (1981), 479-502. For an English version, see arXiv: 1112.2780.

[GLW] Z. Guo, H. Li and C. P. Wang, The second variation formula for Willmore submanifolds in $S^{n}$, Results in Math. 40 (2001), 205-225.

[GT] J. Q. Ge and Z. Z. Tang, Geometry of isoparametric hypersurfaces in Riemannian manifolds, preprint, 2010, arXiv:1006.2577

[GTY] J. Q. Ge, Z. Z. Tang and W. J. Yan, A filtration for isoparametric hypersurfaces in Riemannian manifolds, preprint, 2011, arXiv:1102.1126

[Imm] Stefan Immervoll, On the classification of isoparametric hypersurfaces with four distinct principal curvatures in spheres, Ann. of Math. (2) 168 (2008), no. 3, 1011-1024.

[Mun] H. F. Münzner, Isoparametrische Hyperflächen in Sphären, I and II, Math. Ann., 251 (1980), 57-71 and 256 (1981), 215-232.

[OT] H. Ozeki and M. Takeuchi, On some types of isoparametric hypersurfaces in spheres I and II, Tohoku Math. J., 27 (1975), 515-559 and 28 (1976), 7-55.

$[\mathrm{PW}]$ F. J. Pedit and T. J. Willmore, Conformal geometry, Atti Sem. Mat. Fis. Univ. Modena, 36 (1988), 237-245.

[Sol] B. Solomon, Quartic isoparametric hypersurfaces and quadratic forms, Math. Ann. 293 (1992), 387-398.

[TT] R. Takagi and T. Takahashi, On the principal curvatures of homogeneous hypersurfaces in a sphere, Differential Geometry, in honor of K. Yano, Kinokuniya, Tokyo, 1972.

[TXY] Z. Z. Tang, Y. Q. Xie and W. J. Yan, Gromov-Lawson-Schoen-Yau theory and isoparametric foliations, preprint, 2011, arXiv:1107.5234.

[TY] Z. Z. Tang and W. J. Yan, New examples of Willmore submanifolds in the unit sphere via isoparametric functions, to appear in Ann. Glob. Anal. Geom., (2012), DOI 10.1007/s10455-012-9319-z.

[Wan] C. P. Wang, Moebius geometry of submanifolds in $S^{n}$, Manu. Math., 96 (1998), 517-534.

[Wan'] Q. M. Wang, Isoparametric functions on Riemannian manifolds. I, Math. Ann. 277 (1987), 639-646. 
School of Mathematical Sciences, Laboratory of Mathematics and Complex Systems, Beijing Normal University, Beijing 100875, China

E-mail address: qianchao_1986@163.com

School of Mathematical Sciences, Laboratory of Mathematics and Complex Systems, Beijing Normal University, Beijing 100875, China

E-mail address: zztang@bnu.edu.cn

School of Mathematical Sciences, Laboratory of Mathematics and Complex Systems, Beijing Normal University, Beijing 100875, China

E-mail address: wjyan@mail.bnu.edu.cn 